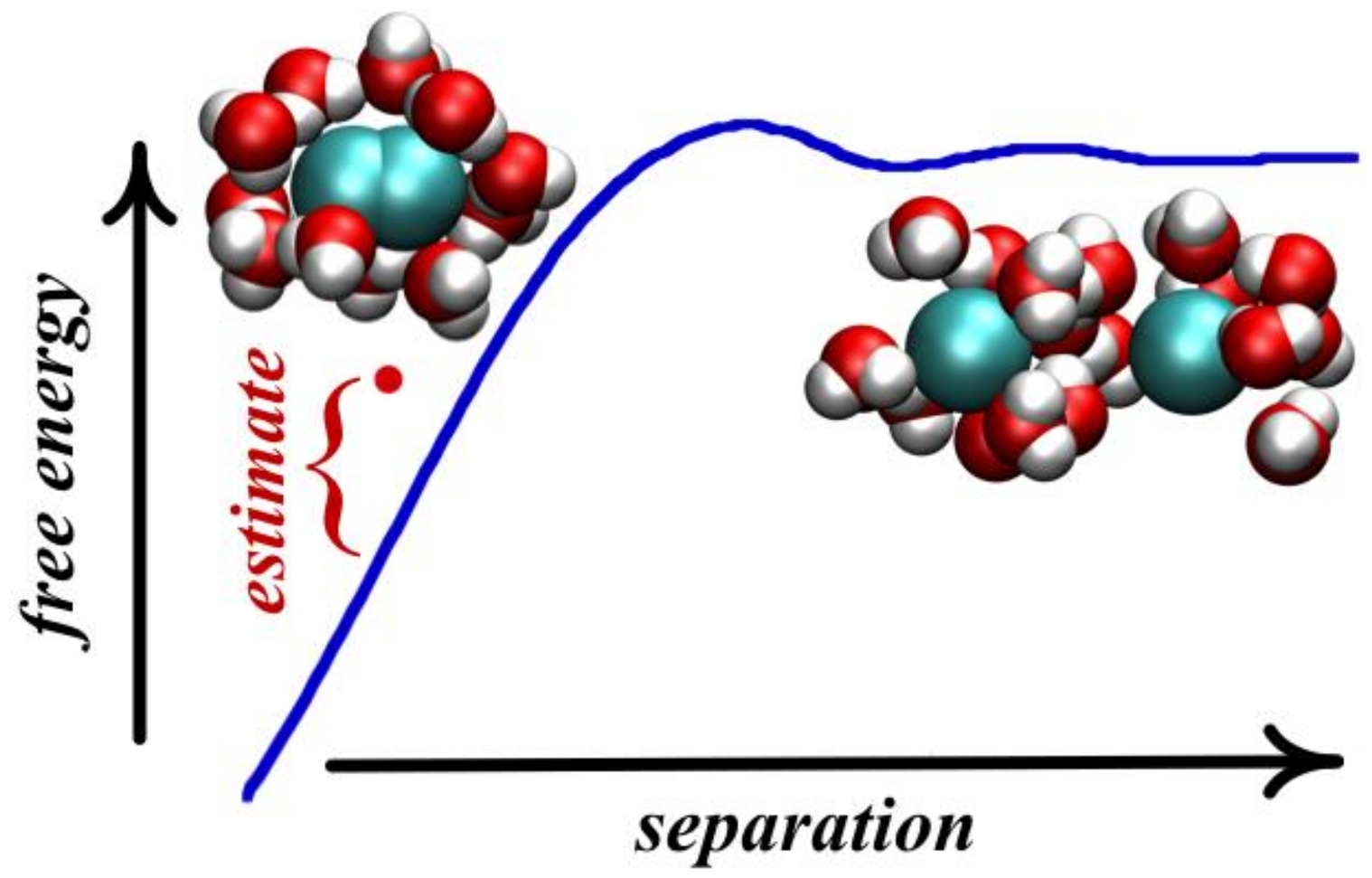

(C) 2016. This manuscript version is made available under the Elsevier user license http://www.elsevier.com/open-access/userlicense/1.0/ 


\title{
Resolving Solvophobic Interactions Inferred from Experimental Solvation Free Energies and Evaluated from Molecular Simulations
}

J. Wesley Barnett ${ }^{1}$, Amna Bhutta ${ }^{2}$, Sarah C. Bierbrier ${ }^{1}$, Natalia da Silva Moura ${ }^{1}$, Henry S. Ashbaugh ${ }^{1, *}$

\begin{abstract}
Ben-Naim estimated the solvent-mediated interaction between methanes based on experimental solvation free energy differences between chemically similar hydrocarbons. Interactions were predicted to be strongest in water, dominated by characteristic entropic gains. We use molecular simulations in combination with an empirical interpolation procedure that bridges interactions from outside methane's excluded volume down to overlap to test BenNaim's estimates. While Ben-Naim's approach captures many distinctive trends, the alchemical differences between methane and a methyl unit play a non-trivial role on the predicted association strength and the sign of enthalpic and entropic components of the interaction free energy in water and ethanol.
\end{abstract}




\section{Introduction}

The substantive attraction between non-polar moieties in water inferred from micellization and protein folding experiments is regularly invoked as a principal driving force for aqueous phase assembly [1, 2]. Experimental examination of hydrophobic interactions in isolation from other water-mediated forces is hampered by the sparing solubility of purely nonpolar species, however, necessitating the inclusion of polar and charged functional groups to increase solute concentrations and raise the observable response to measurable levels. Resultantly, much of our understanding of molecular-level hydrophobic interactions is inferred from experimental studies of the hydration of isolated non-polar species and the assembly of amphipathic species, as well as theoretical and simulation studies of simple non-polar species in water. Computationally, the interaction between methanes, quantified by their potential-of-mean force (PMF) in water, has served as a foundation for understanding hydrophobic interactions [37]. The minimal solubility of even simple solutes like methane makes direct experimental resolution of the PMF by neutron scattering experiments intractable, as noted above. Recent simulation studies have demonstrated that the second-osmotic virial coefficient for simple nonpolar solutes in water, which depends on the integrated PMF, can be reliable evaluated, opening up new avenues for experimental validation [7-10]. In addition, new spectroscopic probes of the hydration shells of hydrophobic moieties and their aggregates have been developed that are providing microscopic structural insights that can potentially bridge the experimental and computational divide $[11,12]$.

In a series of papers from the 1970's [13-17], Ben-Naim proposed an experimental estimate of molecular-scale hydrophobic interactions based on the hydration thermodynamics of small hydrocarbon species. His insight was that solvent-mediated contributions to the PMF are 
molecular simulations appear to over predict the magnitude of the hydrophobic attraction and that this discrepancy needs resolution.

In a recent molecular simulation study of methane interactions in water over a broad range of temperatures and pressures, Ashbaugh and coworkers [7] developed an interpolative ansatz for bridging solvent-mediated interactions evaluated from radial distribution functions passively observed during simulations and the value at direct overlap obtained from particle insertion free energies. Specifically, they proposed the solvent-mediated contribution to the PMF between solutes is described over all separations from overlap out to infinity by the equation

$$
\omega_{\mathrm{mm}}^{j}(r)=\left.f(r) \omega_{\mathrm{mm}}^{j}(r)\right|_{\text {cubic }}+\left.[1-f(r)] \omega_{\mathrm{mm}}^{j}(r)\right|_{\text {sim }} .
$$

The function $\left.\omega_{\mathrm{mm}}^{j}(r)\right|_{\mathrm{sim}}$ is the solvent-mediated contribution to the methane-methane PMF determined from molecular simulations in solvent $j$ following the expression $\left.\omega_{\mathrm{mm}}^{j}(r)\right|_{\mathrm{sim}}=$ $-k T \ln g_{\mathrm{mm}}^{j}(r)-\varphi_{\mathrm{mm}}(r)$ (Here $k T$ is the product of Boltzmann's constant and the temperature, $g_{\mathrm{mm}}^{j}(r)$ is the methane-methane radial distribution function in $j$, and $\varphi_{\mathrm{mm}}(r)$ is the gas phase pair interaction between methanes). The solvent-mediated PMF evaluated from radial distribution functions is accurate only for separations greater than $\sim 3.5 \AA$ as a result of poor/no sampling in the excluded volume regime. The function $\left.\omega_{\mathrm{mm}}^{j}(r)\right|_{\text {cubic }}$ is a fitted cubic function that bridges from the methane overlap value determined from simulation out to separations of $\sim 5$ $\AA$ (evaluated as $\left.\omega_{\mathrm{mm}}^{j}(r)\right|_{\text {cubic }}=\omega_{\mathrm{mm}}^{j}(0)+a r+b r^{2}+c r^{3}$, where $\omega_{\mathrm{mm}}^{j}(0)$ is the value of the PMF at direct methane overlap, and $a, b$, and $c$ are fitted variables smoothly interpolating between overlap and the solvent contribution extracted from the radial distribution function). The function $f(r)$ is a cubic switching function that changes values from 1 to 0 over the range $R_{\text {lower }}$ (= $3.5 \AA)$ to $R_{\text {upper }}(=5 \AA)$ to smoothly transition between $\left.\omega_{\mathrm{mm}}^{j}(r)\right|_{\text {cubic }}$ and $\left.\omega_{\mathrm{mm}}^{j}(r)\right|_{\text {sim }}$. 
Additional information on the development and fitting of eq. (1) can be found in ref. [7]. An advantage of using eq. (1) to obtain the solvent contribution to the PMF over more traditional approaches, like free energy perturbation (e.g., ref. [5]), is that the PMF over a wide range of separations can be determined from the interpolation formula using results from a single simulation rather than from multiple windows.

The capacity of eq. (1) to describe methane interactions over all separations opens up the potential to quantitatively compare Ben-Naim's experimental estimates for the solvent contribution to the PMF in the unobserved overlap regime against molecular simulations. In this paper we compare Ben-Naim's predictions for methane interaction free energies, enthalpies, and entropies in water and ethanol against interpolated molecular simulation results obtained from eq. (1) to assess the fidelity of simulation models at capturing experiment and vice versa.

\section{Simulation Methodology}

Simulations were performed using the GROMACS 5.0 simulation package [20]. Simulations were conducted in the isothermal-isobaric ensemble with the temperature and pressure controlled using the Nosé-Hoover thermostat [21, 22] and Parrinello-Rahman barostat [23], respectively. Simulations were conducted at temperatures from $5^{\circ} \mathrm{C}$ to $65^{\circ} \mathrm{C}$ in $5^{\circ} \mathrm{C}$ increments at 1 bar pressure. We examined solutions of methane, ethane, $\mathrm{p}$ - and o-xylene in water and ethanol. Water was modeled using the TIP4P/2005 potential [24]. Ethanol and the organic solutes were modeled using the TraPPE-United Atom potential [25, 26]. Cross interactions were evaluated using standard Lorentz-Bethelot mixing rules. The methane and methyl group interactions with water's oxygen utilized methane-oxygen and methyl-oxygen Lennard-Jones interaction diameters of $3.436 \AA$ and $3.446 \AA$ and well-depths of $1.023 \mathrm{~kJ} / \mathrm{mol}$ 
and $0.8115 \mathrm{~kJ} / \mathrm{mol}$, respectively, while interactions for xylene's aromatic carbons with water were obtained using Lorentz-Berthelot combining rules [27]. These methane and methyl group interactions were optimized to reproduce the experimental hydration free energies of methane and ethane. We note that while the methane parameters were previously developed for the Hydrophobic Hydration potential [28, 29], the methyl units were re-optimized here to yield improved agreement with experiment. Cross interactions between the hydrocarbons and ethanol were evaluated using Lorentz-Berthelot combining rules. Short range van der Waals interactions were truncated at $10 \AA$ with mean-field energy and pressure dispersion corrections applied beyond the cut-off. Electrostatic interactions were evaluated using particle-mesh Ewald summation [30]. Internal constraints for water were held fixed using the SETTLE [31] algorithm. The LINCS algorithm [32] was used to constrain solute bonds in ethanol and ethane, while SHAKE [33], which is computationally slower than LINCS, was required for the xylene simulations in order to constrain both angles and bonds in the xylene. While the TraPPE-UA potential specifies rigid improper dihedral angles to maintain the planarity of xylene's aromatic ring, these could not be readily implemented in GROMACS. Subsequently, OPLS-UA aromatic dihedral and improper dihedral angles were used for xylene. Equations of motion were evaluated using a 2 fs time step.

In the first set of simulations performed, 10 methanes were placed in simulation boxes of either 1000 waters or 307 ethanols. Simulations were conducted for 100 ns following at least 10 ns of equilibration. Configurations were saved every picosecond for evaluation of methanemethane radial distribution functions and methane overlap free energies. Methane overlap free energies required for eq. (1), i.e., $\omega_{\mathrm{mm}}^{j}(0)$, were determined as the excess hydration free energy difference, in Ben-Naim's standard state, of two overlapping methane less that of two methanes 
in bulk solution. We evaluated these free energies using Widom's test particle insertion method $[34,35]$. In practice the free energy difference is determined as the free energy for inserting a test methane directly on top of a methane in solution with those two methanes not interacting with one another less the free energy of randomly inserting a single methane in bulk solution. In the case of direct insertion on top of a pre-existing methane to evaluate the overlap free energy, insertions were performed over each of the 10 methanes in each saved configuration. For evaluation of the free energy of an individual methane in solution, 10,000 random insertions were attempted over each saved configuration.

In the second set of simulations performed, the excess hydration free energies and free energy differences were evaluated for methane, ethane, and p- and o-xylene at infinite dilution in water and ethanol. In these simulations we considered one solute in a box of 280 to 400 waters (depending on the solute size) or 95 to 151 ethanols. Solvation free energies were evaluated via the multistate Bennett acceptance ratio (MBAR) method [36], using the python alchemical analysis script frontend for pymbar [37] for the computation and uncertainty analysis. Interactions between initial and final states in these free energy calculations were transformed using a coupling parameter $\lambda$ from 0 (initial state) to 1 (final state) in increments of 0.1 for a total of eleven states to evaluate the free energy. Averages for MBAR analysis were conducted over simulations of $10 \mathrm{~ns}$ at each value of $\lambda$ following at least $1 \mathrm{~ns}$ for equilibration. For methane and ethane the full solvation free energy relative to the vacuum in Ben-Naim's standard state was determined for each solute, while the free energy difference between $\mathrm{p}$ - and o-xylene was determined via alchemical shifting of one of the methyl units from the para to ortho position on the aromatic ring relative to a fixed methyl unit. To determine the impact of the interaction differences between methane and the methyl units of ethane, we evaluated free energies for 
transforming ethane's methyl units into methane units using Widom's insertion technique [34, 35]. We refer to this ethane comprised of two bonded methanes as m/ethane below.

The free energies and PMFs evaluated from our simulations described above were broken down into their entropic and enthalpic components by fitting their temperature dependencies to the function

$$
g=\alpha+\beta\left(T-T_{0}\right)+\gamma T \ln \left(T / T_{0}\right)
$$

which assumes a constant heat capacity. In this expression $g$ is either the solute's excess solvation free energy or the PMF, $\omega_{\mathrm{mm}}^{j}(r)$, while $T_{0}$ is a reference temperature taken here to be $298.15 \mathrm{~K}\left(25^{\circ} \mathrm{C}\right)$. The parameters $\alpha, \beta$, and $\gamma$ are fitting constants in the case of solvation free energies, or separation-dependent fitting functions in the case of the PMF. Entropies and enthalpies were evaluated from the temperature derivatives of eq. (2), i.e., $s=-\partial g /\left.\partial T\right|_{P}$ and $h=\partial(g / T) /\left.\partial(1 / T)\right|_{P}$.

\section{Results and Discussion}

The solvent-mediated interaction between two methanes in water at $25^{\circ} \mathrm{C}$ is reported in Figure 1. The portion of the PMF determined directly from the radial distribution function evaluated from molecular simulation, $\left.\omega_{\mathrm{mm}}^{\mathrm{wat}}(r)\right|_{\text {sim }}$, extend from bulk solution down to a separation of $3.15 \AA$. Barring the sharp down turn in $\left.\omega_{\mathrm{mm}}^{\mathrm{wat}}(r)\right|_{\text {sim }}$ below $r<3.25 \AA$, which results from poor sampling in this region, the PMF evaluated from simulation at small separations points down towards the methane overlap limit, $\omega_{\mathrm{mm}}^{\mathrm{wat}}(0)$, determined from methane insertion calculations. Eq. (1) smoothly interpolates the divide between complete overlap and the simulation accessible regime, providing a reasonable description of the water-mediated methane interaction over all separations (Figure 1). Based on the quality of the description shown here 
and our previous investigations [7], we have confidence that eq. (1) provides an accurate description of the solvent-mediated between methanes across all separations.

The temperature dependence of the solvent-mediated methane interaction in water and ethanol was subsequently determined by fitting the results of eq. (1) determined over the temperature range $5^{\circ} \mathrm{C}$ to $65^{\circ} \mathrm{C}$ to eq. (2). Taking appropriate temperature derivatives of the $\mathrm{PMF}$, the free energy, enthalpy, and entropy of methane association in water and ethanol at $25^{\circ} \mathrm{C}$ are reported in Figure 2. While the methane PMFs in both water and ethanol are attractive down into the excluded volume regime (Figure $2 \mathrm{a}$ and $\mathrm{d}$ ), the attraction is strongest in water, consistent with the idea that water promotes assembly. While the enthalpy of association is attractive in both solvents (Figure $2 \mathrm{~b}$ and e), the enthalpic attraction is stronger in alcohol than it is in water. In water the methane interaction entropy is attractive down into the excluded volume region (Figure 2c), while in ethanol the interaction entropy is repulsive (Figure 2f). This interaction entropy disparity between water and organic solvents has been taken as a hydrophobic interaction signature.

Ben Naim suggested that the solvent-mediated interaction between methanes at a fixed separation of $1.54 \AA$ (the bond length of ethane) could be estimated from the difference between the excess chemical potential of ethane in $j=$ water or ethanol less twice that of methane

$$
\omega_{\mathrm{mm}}^{j}(1.54 \AA) \approx \Delta \mu_{\mathrm{ethane}}^{\mathrm{ex}, j}=\mu_{\mathrm{ethane}}^{\mathrm{ex}, j}-2 \mu_{\mathrm{methane}}^{\mathrm{ex}, j}
$$

This approximation effectively assumes that ethane's two methyl units are equivalent to two methanes. This relationship can be made exact in our simulations by replacing the free energy of ethane for that of m/ethane (that is an ethane with the methyl units alchemically transformed into methanes) in eq. (3) (i.e., $\left.\omega_{\mathrm{mm}}^{j}(1.54 \AA)=\Delta \mu_{\mathrm{m} / \mathrm{ethane}}^{\mathrm{ex}, j}=\mu_{\mathrm{m} / \mathrm{ethane}}^{\mathrm{ex}, j}-2 \mu_{\mathrm{methane}}^{\mathrm{ex}, j}\right)$. The excess solvation thermodynamic properties for methane, ethane, and m/ethane in water and ethanol at 
$25^{\circ} \mathrm{C}$ needed to estimate the methane interaction thermodynamics at $r=1.54 \AA$ following BenNaim's prescription are reported in Table 1. The simulation results for methane and ethane are in excellent agreement with the available experimental numbers [15, 38, 39], giving us confidence in the fidelity of the computational models used for describing solvation.

The interaction thermodynamics between methanes evaluated from eq. (3) are reported in Table 2 and compared against the solvent-mediated PMFs in Figure 2. While we find that the interaction free energies predicted from ethane's solvation free energy, $\Delta \mu_{\mathrm{ethane}}^{\mathrm{ex}, j}$, are attractive in both water and ethanol, the predicted interaction free energies are more positive than the solventmediated PMF at $r=1.54 \AA$ (Figure $2 \mathrm{a}$ and $2 \mathrm{~d}$ ). The free energies determined from m/ethane's solvation free energy, $\Delta \mu_{\mathrm{m} / \mathrm{ethane}}^{\mathrm{ex}, j}$ are in quantitative agreement with the interpolated PMF obtained from eq. (1) in the excluded volume regime (Figure $2 \mathrm{a}$ and $2 \mathrm{~d}$ ), however, indicating that the alchemical differences between methane and a methyl group is sufficient to account for BenNaim's prediction of a lower solvophobic interaction free energy.

Similar to the interaction free energies, the interaction enthalpies in either solvent predicted by eq. (3) from ethane solvation data are more positive and repulsive than those obtained from the solvent-mediated PMF (Figure $2 b$ and $2 e$ ). The magnitude of the enthalpy difference is between 8 to $9 \mathrm{~kJ} / \mathrm{mol}$ at room temperature (Table 2). This difference is significant enough in water such that the predicted methane interaction enthalpy in water is positive, while we observe a negative interaction enthalpy from the PMF (Figure 2b) and our results from m/ethane hydration (Table 2).

In difference to the enthalpy, the interaction entropies predicted from Ben-Naim's approximation are more attractive than that obtained from the solvent-mediated PMF (Figure 2c and 2f). The magnitude of this difference accounts for a 2 to $3 \mathrm{~kJ} / \mathrm{mol}$ lower entropic 
contribution predicted by eq. (3) in both water and ethanol (Table 2). While smaller in magnitude than the enthalpy, this difference is significant enough in the case of ethanol-mediated interactions that the entropy predicted from Ben-Naim's approximation is attractive while that obtained from the PMF is repulsive (Figure 2f), in agreement with the results obtained from m/ethane solvation (Table 2).

While their diameters are practically the same, the interaction well-depth of methane is deeper than that of an ethane methyl unit in the simulation models used. This observation suggests the reason Ben-Naim's approximation predicts a more repulsive enthalpy is the result of attractive interaction differences of a methyl unit and methane with the solvent. Assuming minimal changes in the solvent structure and volume for alchemically changing ethane's methyl units into methanes, the excess solvation free energy between m/ethane and ethane is approximately given as

$$
\mu_{\mathrm{m} / \mathrm{ethane}}^{\mathrm{ex}, j} \approx \mu_{\mathrm{ethane}}^{\mathrm{ex}, j}+\left\langle\Delta \varphi_{\mathrm{e} 2 \mathrm{~m}}\right\rangle_{\mathrm{ethane}},
$$

where the perturbation contribution $\left\langle\Delta \varphi_{\mathrm{e} 2 \mathrm{~m}}\right\rangle_{\mathrm{ethane}}$ is the potential energy difference between treating ethane's methyl units as united-atom methanes versus united-atom methyl units averaged over configurations generated from our ethane simulations. The solvation free energies, enthalpies, and entropies at $25^{\circ} \mathrm{C}$ determined from eq. (4) are in excellent quantitative agreement with the exact values for the m/ethane reported in Table 1. Resultantly the perturbation corrected interaction thermodynamic predictions are in harmony with those determined from the solventmediated PMF (Table 2). We can conclude that the difference between the Ben-Naim's predicted interaction thermodynamics and the solvent-mediated PMF within the excluded volume regime results from interaction differences between a methane and methyl unit with the solvent, and not from changes in the solvent structure around methanes or methyls held together at a $1.54 \AA$ 
separation. While eq. (4) only relies on energetic differences between methyl units and methane, the temperature dependence of this interaction energy difference is sufficient to account for the entropic differences between that predicted from Ben-Naim's estimate and the simulation PMF.

It has been suggested that the solvation free energy difference between o- and p-xylene provides an approximation to the solvent-mediated interaction between methanes at a separation of $2.94 \AA$ (the distance between the methyl units on o-xylene), not as deep into the excluded volume regime as determined from ethane $[16,19]$. The solvation thermodynamic differences between the xylene isomers, $\Delta \theta_{\mathrm{xylene}}^{\mathrm{ex}, j}=\theta_{\mathrm{o}-\mathrm{xylene}}^{\mathrm{ex}, j}-\theta_{\mathrm{p}-\mathrm{xylene}}^{\mathrm{ex}, j}$, evaluated from our alchemical change simulations are reported in Table 2. While we were unable to find experimental results for xylene solvation in ethanol or the temperature derivatives of the free energy in water, the experimental and simulation solvation free energy differences in water are in excellent agreement. The xylene difference free energies in water and ethanol determined from simulation indicate that the methyl group interaction is favorable and strongest in water. Moreover, the simulations indicate that the xylene methyl unit interaction is entropically favorable in water and unfavorable in ethanol. When we compare the methyl unit interaction thermodynamics predicted from the p-/o-xylene difference calculation against the solvent-mediated methane-methane PMFs, however, the comparison is weak (Figure 2). Specifically, compared to the methanemethane interaction thermodynamics the results estimated from xylene solvation are negligible. It may be noted that the difference between $\mathrm{p}$ - and o-xylene more accurately represents the free energy difference of bringing a methyl unit from a separation of $5.86 \AA$ (the methyl unit separation in p-xylene) to $2.94 \AA$. Examining the methane-methane PMFs, however, we find that the PMF values at $5.86 \AA$ are significantly closer to zero than at $2.94 \AA$, suggesting the difference between these two separations is comparable to the free energy of bringing the two 
carbons together from infinity. We have also performed free energy perturbation calculations to transform xylene's methyl units into methanes, however, this calculation barely changed the results obtained with the methyl group interactions.

We attribute the lower magnitude xylene estimates of the interaction free energy to the incomplete solvation of the methyl units, whose solvation is blocked by xylene's aromatic ring barring the formation of a complete solvation shell about the methyl units for either isomer. Indeed, if we compare the change in the solvent accessible surface area [40] between xylene isomers versus the change in area between bringing two methanes to the methyl unit separation in ortho-xylene we find significant differences. The area difference between ortho- and paraxylene is $-8 \AA^{2}$, while that for brining methanes together is $-76 \AA^{2}$ (The van der Waals radii of water, $\mathrm{CH}_{3 / 4}$, and the aromatic carbons were assumed to be $1.4 \AA, 1.9 \AA$, and $1.7 \AA$, respectively in this calculation). The difference in solvent exposure for xylene is $10 \%$ that of two methanes, suggesting the solvent contribution to the PMF estimated from xylene will be negligible compared to that of two fully hydrated methanes.

\section{Conclusion}

In conclusion, using a previously developed empirical bridge describing the solventmediated interaction between methanes outside their mutually excluded volume down to complete overlap, we have constructed a description for the solvent-mediated PMF over all separations using information garnered simply from molecular simulations of methanes in solution. Comparing the interaction thermodynamics determined from this empirical bridge against earlier experimental estimates of the interaction between methanes at separations of 1.54 $\AA$ and $2.94 \AA$, obtained from the solvation free energy difference between ethane and methane as 
well as p- and o-xylene, we find distinct differences between these two approaches. Specifically, while Ben-Naim's experimental estimate correctly predicts stronger interactions in water versus ethanol, it under predicts the magnitude of the solvent-mediated attraction in both solvents. Moreover, Ben-Naim's estimate incorrectly predicts methane interactions at $1.54 \AA$ (the carboncarbon bond length of ethane) are enthalpically unfavorable in water and entropically favorable in ethanol. The difference between Ben-Naim's estimates based on the differences between ethane and methane and the present simulation results were shown to be a result of alchemical distinctions between the methyl units of ethane and methane. When these are taken into account following a simple linear perturbation theory approach to account for the direct interaction difference between ethane's methyl units and methane with the solvent, Ben-Naim's estimate can be reconciled with methane's potential-of-mean force. While interaction estimates made based on the solvation differences between xylene isomers predict stronger interactions in water compared to ethanol, the magnitude of the estimated interactions are weaker than those observed for methane's in solution largely due to the incomplete solvation of the methyl units blocked by xylene's aromatic ring.

We believe these are the first results bringing these two distinct methods for evaluating methane hydrophobic interactions into harmony with one another. Once alchemical differences are taken into account, the agreement between Ben-Naim's estimates and our simulations gives us confidence that simulations using models optimized to reproduce solvation thermodynamic provide an accurate representation of the solvent-mediated interactions.

\section{Acknowledgements}


This work has benefited from numerous conversations with Prof. Dor Ben-Amotz (Purdue). We gratefully acknowledge financial support from a Louisiana Board of Regents Graduate Research Fellowship (JWB) and from the NSF (OIA-1430280). 


\section{Figure Captions}

Figure 1. Water-mediated contribution to the methane-methane potential-of-mean force at a temperature of $25^{\circ} \mathrm{C}$. Results are shown for the potential-of-mean force obtained directly from the simulation radial distribution function, $\left.\omega_{\mathrm{mm}}^{\text {wat }}(r)\right|_{\text {sim }}$, the free energy at methane overlap $\omega_{\mathrm{mm}}^{\mathrm{wat}}(0)$, and for the fit of eq. (1) bridging the simulation and overlap free energies, $\omega_{\mathrm{mm}}^{\mathrm{Wat}}(r)$. The symbols are defined in the figure legend.

Figure 2. Free energy (a and d), enthalpy (b and e), and entropy (c and $\mathbf{f}$ ) of methane association in water $(\mathbf{a}-\mathbf{c})$ and ethanol $(\mathbf{d}-\mathbf{f})$ at $25^{\circ} \mathrm{C}$ and 1 bar as a function of separation from direct overlap ( $r=0$ ) to a separation of $12 \AA$. The figure symbols are defined in the legend on the right hand side of the figure. The entropies and enthalpies were determined by fitting the PMFs and solvation free energies to eq. (2) and taking the appropriate temperature derivatives. 


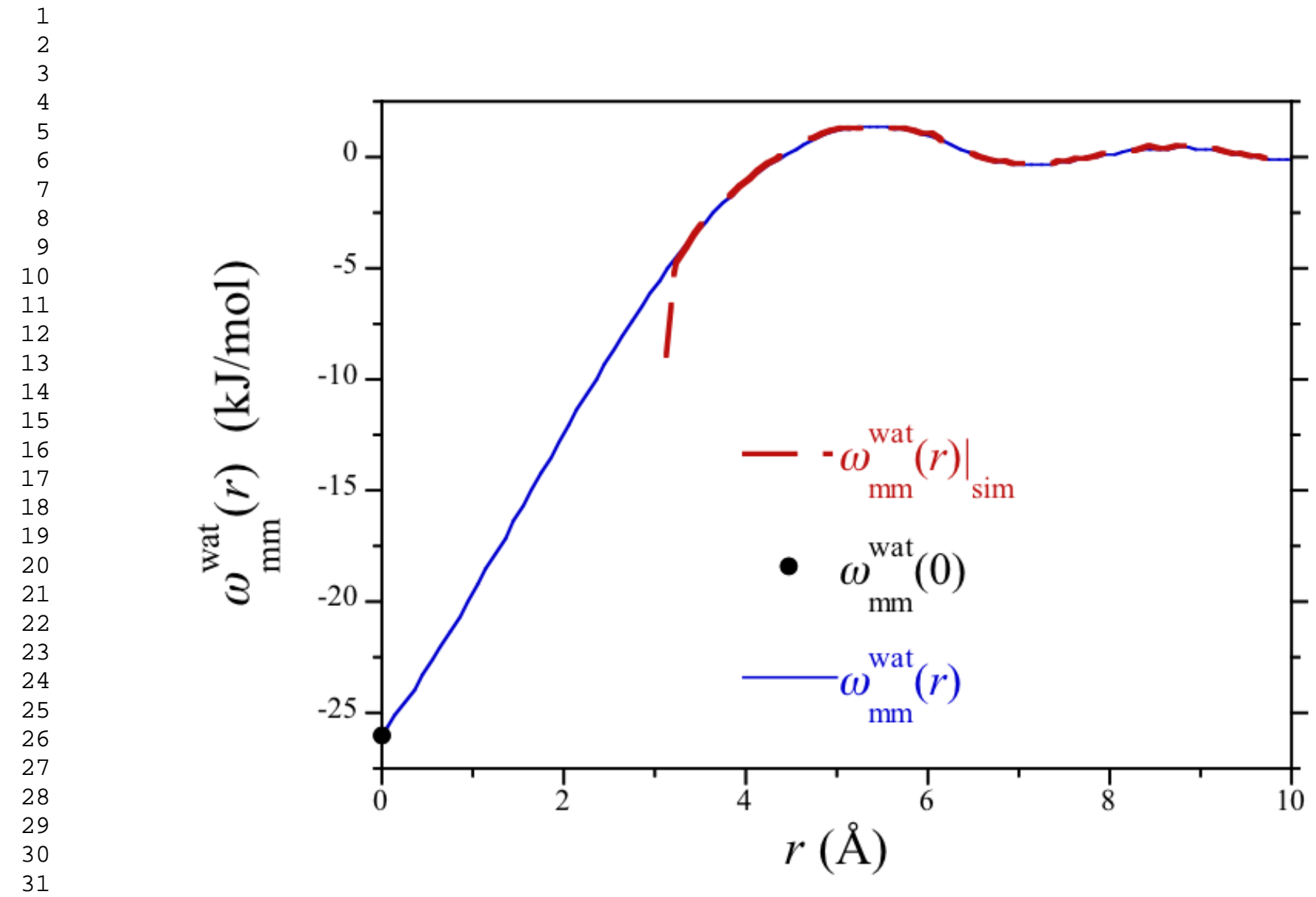

Figure 1. 

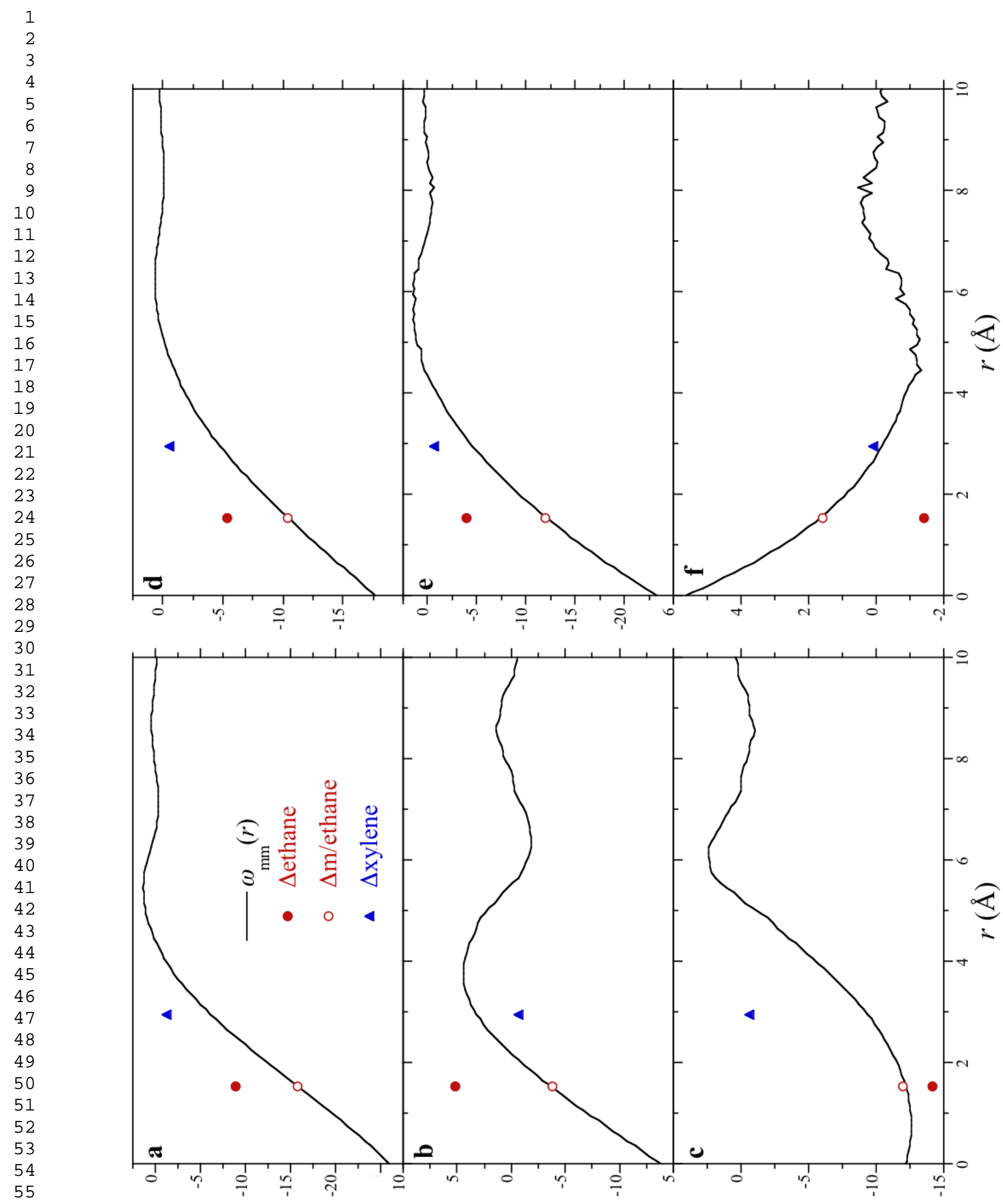

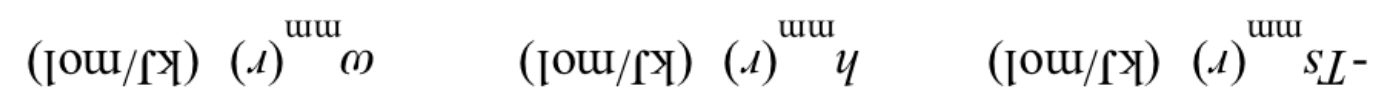

Figure 2. 
Table 1. Excess solvation thermodynamic properties for hydrocarbons in water and ethanol at $25^{\circ} \mathrm{C}$ and 1 bar from simulation and experiment. Simulation error bars reported in parentheses correspond to one standard deviation. Experimental results were obtained from refs. [15, 38, 39].

\begin{tabular}{|c|c|c|c|c|c|c|}
\hline & & water & & & ethanol & \\
\hline & $\begin{array}{c}\mu_{i}^{\text {ex,wat }} \\
(\mathrm{kJ} / \mathrm{mol})\end{array}$ & $\begin{array}{c}h_{i}^{\text {ex, wat }} \\
(\mathrm{kJ} / \mathrm{mol})\end{array}$ & $\begin{array}{c}-T s_{i}^{\mathrm{ex}, \mathrm{wat}} \\
(\mathrm{kJ} / \mathrm{mol})\end{array}$ & $\begin{array}{c}\mu_{i}^{\mathrm{ex}, \mathrm{alc}} \\
(\mathrm{kJ} / \mathrm{mol})\end{array}$ & $\begin{array}{c}h_{i}^{\text {ex,alc }} \\
(\mathrm{kJ} / \mathrm{mol})\end{array}$ & $\begin{array}{c}-T s_{i}^{\text {ex,alc }} \\
(\mathrm{kJ} / \mathrm{mol})\end{array}$ \\
\hline $\begin{array}{l}\text { simulation } \\
\text { methane }\end{array}$ & $8.30(0.02)$ & $-11.22(0.44)$ & $19.52(0.44)$ & $1.51(0.01)$ & $-2.15(0.24)$ & $3.66(0.24)$ \\
\hline ethane & $7.64(0.02)$ & $-17.27(0.52)$ & $24.91(0.52)$ & $-2.38(0.02)$ & $-8.30(0.29)$ & $5.92(0.29)$ \\
\hline $\mathrm{m} /$ ethane & $0.83(0.03)$ & $-26.24(0.43)$ & $27.06(0.42)$ & $-7.40(0.01)$ & $-16.30(0.31)$ & $8.90(0.31)$ \\
\hline ethane+perturbation & $0.93(0.05)$ & $-26.18(0.55)$ & $27.12(0.58)$ & $-7.82(0.03)$ & $-16.71(0.44)$ & $8.88(0.43)$ \\
\hline $\begin{array}{l}\text { experiment } \\
\text { methane }\end{array}$ & 8.39 & -11.50 & 19.89 & 1.58 & -2.81 & 4.39 \\
\hline ethane & 7.67 & -17.47 & 25.14 & -2.50 & -9.44 & 6.94 \\
\hline
\end{tabular}


Table 2. Estimates for solvent-mediated methane-methane interaction thermodynamics determined from excess solvation properties at $25^{\circ} \mathrm{C}$ and 1 bar following Ben-Naim's difference approach using both simulation and experimental data. Simulation error bars reported in parentheses correspond to one standard deviation. Experimental results were obtained from refs. $[15,38,39]$.

\begin{tabular}{|c|c|c|c|c|c|c|}
\hline & \multicolumn{3}{|c|}{ water } & \multicolumn{3}{|c|}{ ethanol } \\
\hline & $\begin{array}{l}\Delta \mu_{i}^{\mathrm{ex}, \mathrm{wat}} \\
(\mathrm{kJ} / \mathrm{mol})\end{array}$ & $\begin{array}{l}\Delta h_{i}^{\text {ex,wat }} \\
(\mathrm{kJ} / \mathrm{mol})\end{array}$ & $\begin{array}{c}-T \Delta s_{i}^{\mathrm{ex}, \mathrm{wat}} \\
(\mathrm{kJ} / \mathrm{mol})\end{array}$ & $\begin{array}{l}\Delta \mu_{i}^{\text {ex,alc }} \\
(\mathrm{kJ} / \mathrm{mol})\end{array}$ & $\begin{array}{l}\Delta h_{i}^{\text {ex,alc }} \\
(\mathrm{kJ} / \mathrm{mol})\end{array}$ & $\begin{array}{c}-T \Delta s_{i}^{\text {ex alc }} \\
(\mathrm{kJ} / \mathrm{mol})\end{array}$ \\
\hline $\begin{array}{l}\text { simulation } \\
\text { ethane }\end{array}$ & $-8.96(0.05)$ & $5.17(0.77)$ & $-14.14(0.79)$ & $-5.40(0.04)$ & $-4.00(0.39)$ & $-1.40(0.39)$ \\
\hline m/ethane & $-15.78(0.06)$ & $-3.80(0.97)$ & $-11.98(0.96)$ & $-10.42(0.03)$ & $-12.00(0.36)$ & $1.58(0.35)$ \\
\hline ethane+perturbation & $-15.67(0.07)$ & $-3.74(0.79)$ & $-11.93(0.75)$ & $-10.84(0.03)$ & $-12.41(0.51)$ & $1.56(0.50)$ \\
\hline xylene (ortho-para) & $-1.31(0.02)$ & $-0.69(0.15)$ & $-0.62(0.15)$ & $-0.53(0.01)$ & $-0.63(0.07)$ & $0.10(0.07)$ \\
\hline $\begin{array}{l}\text { experiment } \\
\text { ethane }\end{array}$ & -9.10 & 5.53 & -14.63 & -5.65 & -3.81 & -1.84 \\
\hline xylene (ortho-para) & -1.27 & N/A & N/A & N/A & N/A & N/A \\
\hline
\end{tabular}




\section{References.}

[1] C. Tanford, The Hydrophobic Effect: Formation of Micelles and Biological Membranes, second ed., John Wiley and Sons, New York, 1980.

[2] W. Blokzijl, J.B.F.N. Engberts, Hydrophobic effects. Opinions and facts, Angew. Chem. Int. Ed., 32 (1993) 1545-1579.

[3] L.R. Pratt, D. Chandler, Theory of the Hydrophobic Effect, J. Chem. Phys., 67 (1977) 36833704.

[4] C. Pangali, M. Rao, B.J. Berne, Monte-Carlo Simulation of the Hydrophobic Interaction, J. Chem. Phys., 71 (1979) 2975-2981.

[5] D.E. Smith, A.D.J. Haymet, Free-Energy, Entropy, and Internal Energy of Hydrophobic Interactions - Computer Simulations, J. Chem. Phys., 98 (1993) 6445-6454.

[6] G. Hummer, S. Garde, A.E. Garcia, A. Pohorille, L.R. Pratt, An Information Theory Model of Hydrophobic Interactions, Proc. Natl. Acad. Sci. U. S. A., 93 (1996) 8951-8955.

[7] H.S. Ashbaugh, K. Weiss, S.M. Williams, B. Meng, L.N. Surampudi, Temperature and Pressure Dependence of Methane Correlations and Osmotic Second Virial Coefficients in Water, J. Phys. Chem. B, 119 (2015) 6280-6294.

[8] K. Koga, Osmotic Second Virial Coefficient of Methane in Water, J. Phys. Chem. B, 117 (2013) 12619-12624.

[9] M.I. Chaudhari, S.A. Holleran, H.S. Ashbaugh, L.R. Pratt, Molecular-Scale Hydrophobic Interactions between Hard-Sphere Reference Solutes are Attractive and Endothermic, Proc. Natl. Acad. Sci. U. S. A., 110 (2013) 20557-20562.

[10] M.I. Chaudhari, D. Sabo, L.R. Pratt, S.B. Rempe, Hydration of $\operatorname{Kr}(\mathrm{aq})$ in Dilute and Concentrated Solutions, J. Phys. Chem. B, (2015) 9098-9102.

[11] D.S. Wilcox, B.M. Rankin, D. Ben-Amotz, Distinguishing Aggregation from Random Mixing in Aqueous t-Butyl Alcohol Solutions, Faraday Discuss., 167 (2013) 177-190.

[12] B.M. Rankin, D. Ben-Amotz, S.T. van der Post, H.J. Bakker, Contacts Between Alcohols in Water Are Random Rather than Hydrophobic, J. Phys. Chem. Lett., 6 (2015) 688-692.

[13] A. Ben-Naim, Statistical Mechanical Study of Hydrophobic Interaction. I. Interaction between Two Identical Nonpolar Solute Particles, J. Chem. Phys., 54 (1971) 1387-1404.

[14] A. Ben-Naim, J. Wilf, M. Yaacobi, Hydrophobic Interaction in Light and Heavy Water, Journal of Physical Chemistry, 77 (1972) 95-102.

[15] M. Yaacobi, A. Ben-Naim, Solvophobic Interaction, Journal of Physical Chemistry, 78 (1974) 175-178.

[16] A. Ben-Naim, J. Wilf, Direct Measurement of Intra-Molecular Hydrophobic Interactions, J. Chem. Phys., 70 (1979) 771-777.

[17] A. Ben-Naim, Hydrophobic Interactions, Plenum Press, New York, 1980.

[18] J.Z. Wu, J.M. Prausnitz, Pairwise-Additive Hydrophobic Effect for Alkanes in Water, Proc. Natl. Acad. Sci. U. S. A., 105 (2008) 9512-9515.

[19] D. Ben-Amotz, Hydrophobic Ambivalence: Teetering on the Edge of Randomness, J. Phys. Chem. Lett., 6 (2015) 1696-1701.

[20] B. Hess, C. Kutzner, D. van der Spoel, E. Lindahl, GROMACS 4: Algorithms for Highly Efficient, Load-Balanced, and Scalable Molecular Simulation, Journal of Chemical Theory and Computation, 4 (2008) 435-447.

[21] S. Nosé, A Unified Formulation of the Constant Temperature Molecular-Dynamics Methods, J. Chem. Phys., 81 (1984) 511-519. 

Review A, 31 (1985) 1695-1697.

[23] M. Parrinello, A. Rahman, Polymophic Transitions in Single-Crystals - A New MolecularDynamics Method, J. Appl. Phys., 52 (1981) 7182-7190.

[24] J.L.F. Abascal, C. Vega, A general purpose model for the condensed phases of water: TIP4P/2005, J. Chem. Phys., 123 (2005) 234505.

[25] M.G. Martin, J.I. Siepmann, Transferable potentials for phase equilibria. 1. United-atom description of n-alkanes, J. Phys. Chem. B, 102 (1998) 2569-2577.

[26] C.D. Wick, M.G. Martin, J.I. Siepmann, Transferable potentials for phase equilibria. 4. United-atom description of linear and branched alkenes and alkylbenzenes, J. Phys. Chem. B, 104 (2000) 8008-8016.

[27] M.P. Allen, D.J. Tildesley, Computer simulation of liquids, Oxford University Press, Oxford, UK, 1987.

[28] H.S. Ashbaugh, L.X. Liu, L.N. Surampudi, Optimization of linear and branched alkane interactions with water to simulate hydrophobic hydration, J. Chem. Phys., 135 (2011) 054510.

[29] H.S. Ashbaugh, N.J. Collett, H.W. Hatch, J.A. Staton, Assessing the thermodynamic signatures of hydrophobic hydration for several common water models, J. Chem. Phys., 132 (2010) 7.

[30] T. Darden, D. York, L. Pedersen, Particle Mesh Ewald: An N·log(N) Method for Ewald Sums in Large Systems, J. Chem. Phys., 98 (1993) 10089-10092.

[31] S. Miyamoto, P.A. Kollman, SETTLE - An Analytical Version of the Shake and Rattle Algortihm for Rigid Water Models, Journal of Computational Chemistry, 13 (1992) 952-962.

[32] B. Hess, H. Bekker, H.J.C. Berendsen, J. Fraaije, LINCS: A linear constraint solver for molecular simulations, Journal of Computational Chemistry, 18 (1997) 1463-1472.

[33] J.P. Ryckaert, G. Ciccotti, H.J.C. Berendsen, Numerical-Integration of Cartesian Equations of Motion os a System with Constraints - Molecular Dynamics of n-Alkanes, Journal of Computational Physics, 23 (1977) 327-341.

[34] B. Widom, Some topics in the theory of fluids, J. Chem. Phys., 39 (1963) 2808-2812.

[35] B. Widom, Potential-distribution theory and the statistical-mechanics of fluids, Journal of Physical Chemistry, 86 (1982) 869-872.

[36] M.R. Shirts, J.D. Chodera, Statistically optimal analysis of samples from multiple equilibrium states, J. Chem. Phys., 129 (2008) 124105.

[37] P.V. Klimovich, M.R. Shirts, D.L. Mobley, Guidelines for the analysis of free energy calculations, Journal of Computer-Aided Molecular Design, 29 (2015) 397-411.

[38] N.V. Plyasunova, A.V. Plyasunov, E.L. Shock, Database of thermodynamic properties for aqueous organic compounds, Int. J. Thermophys., 25 (2004) 351-360.

[39] E.L. Shock, ORganic Compounds HYDration Properties Database, http://orchyd.asu.edu/, August 15, 2016.

[40] B. Lee, F.M. Richards, The interpretation of protein structures: Estimation of static accessibility, Journal of Molecular Biology, 55 (1971) 379-400. 